\title{
Inhibition of the cytotoxic effect of Clostridium difficile in vitro by Clostridium butyricum MIYAIRI 588 strain
}

Correspondence

Timothy D. H. Woo

tdhw3@churchillians.net

Received 14 April 2011

Accepted 17 June 2011

\author{
Timothy D. H. Woo, ${ }^{1}$ Kentaro Oka, ${ }^{2}$ Motomichi Takahashi, ${ }^{2}$ Fuhito Hojo, ${ }^{1}$ \\ Takako Osaki, ${ }^{1}$ Tomoko Hanawa, ${ }^{1}$ Satoshi Kurata, ${ }^{1}$ Hideo Yonezawa ${ }^{1}$ \\ and Shigeru Kamiya ${ }^{1}$ \\ ${ }^{1}$ Department of Infectious Diseases, Kyorin University School of Medicine, Shinkawa 6-20-2,
Mitaka, Tokyo 181-8611, Japan
${ }^{2}$ Miyarisan Pharmaceuticals, Hanishina-gun, Sakaki-machi 102-15, Nagano 389-0682, Japan
}

In contrast to most modern pharmaceuticals, probiotics are used in many parts of the world with little or no research data on the complex system of interactions that each strain may elicit in the human body. Research on probiotics has recently become more significant, as probiotics have begun to be prescribed by clinicians as an alternative for some gut infections, especially when antibiotics are contraindicated. This study attempted to elucidate the inhibitory interaction between the Japanese probiotic strain Clostridium butyricum MIYAIRI 588 (CBM588) and the hospital pathogen Clostridium difficile, which is responsible for a large proportion of antibioticassociated diarrhoea and colitis. CBM588 has previously shown effectiveness against $C$. difficile in vivo, and here it was found that the toxicity of $C$. difficile in in vitro co-culture with CBM588 was greatly decreased or absent. This was dependent on the inoculation ratio and was not accounted for by the small degree of growth and mRNA inhibition observed. CBM588 and its cell-free supernatant also had no effect on toxin already secreted into the culture medium, and culture of the two strains separated by a semi-permeable membrane resulted in loss of the inhibition.

Therefore, it was concluded that the detoxification probably occurred by the inhibition of toxin protein production and that this required close proximity or contact between the two species. The low-pH conditions caused by organic acid secretion were also observed to have inhibitory effects on C. difficile growth, metabolism and toxicity.

\section{INTRODUCTION}

Clostridium difficile-associated disease (CDAD) continues to be a problem in hospitals around the world. Recent UK statistics show an incidence in patients aged over 65 years of over 24000 cases year $^{-1}$ at an annual cost of around $£ 103$ million (Wilcox et al., 1996). Although the primary cost of disease is increased costs of patient stay and treatment, mortality is also significant, particularly among the elderly (Pépin et al., 2004; Henrich et al., 2009). Infection is usually treated with metronidazole or vancomycin, as most isolates remain susceptible to these antibiotics (Huang et al., 2009), but the most efficient measure to curb C. difficile appears to be adequate prophylaxis rather than treatment.

As the growth of $C$. difficile is purported to be due to alterations of the gut flora by antibiotics that normally prevent C. difficile spores from germinating (Borriello \& Barclay, 1986; Preidis \& Versalovic, 2009), probiotics have

Abbreviations: CBM588, Clostridium butyricum MIYAIRI 588 strain; CDAD, C. difficile-associated disease; SCFA, short-chain fatty acid. been proposed to help rectify the imbalance (Engelbrektson et al., 2009). However, the evidence for probiotics in the treatment of CDAD is not conclusive. Several clinical trials have shown positive effects of various probiotics commonly Lactobacillus rhamnosus GG (Segarra-Newnham, 2007) and Saccharomyces boulardii (McFarland, 2010) - but many trials are inconclusive or show marginal effects. Common problems are inconsistencies with dosing, outcome measure and treatment period, as well as dropouts during follow-up, preventing general conclusions of efficacy from being drawn (McFarland, 2009).

Clostridium butyricum MIYAIRI 588 (CBM588) is a Japanese probiotic strain isolated from a patient in 1933 that is currently sold commercially. Like C. difficile, CBM588 is a Gram-positive obligate anaerobe, but it grows faster and utilizes a wider range of substrates. It produces large quantities of short-chain fatty acids (SCFAs) (deVos et al., 2009), especially butyrate, and an active bacteriocin (Nakanishi \& Tanaka, 2010); it also sporulates, allowing it to resist antibiotics that may disrupt other gut flora. It has 
previously shown effectiveness in the prophylaxis of CDAD in a mouse model (Kamiya et al., 1997) and in a clinical trial for prevention of antibiotic-associated diarrhoea in paediatric patients (Seki et al., 2003). In another clinical trial, CBM588 significantly reduced the incidence of detection of C. difficile toxin in the stools of patients taking Helicobacter pylori-eradication therapy (Imase et al., 2008). All of these data make CBM588 an interesting candidate for CDAD prophylaxis and so, in this study, we attempted to investigate this inhibitory effect in vitro.

\section{METHODS}

Bacterial strains and culture conditions. C. difficile VPI 10463, the reference strain against which new toxinotypes are compared, was used in this study. This strain produces large amounts of toxin that are easily measurable by Western blot. Clinical isolates including TMG-10, KY-8, KY-34, KY-104 and SLC were isolated in this laboratory. Additionally, KZ-1692, a non-toxigenic strain, was isolated previously (Nakamura et al., 1980). CBM588 was kindly provided by Miyarisan Pharmaceutical Co. Strains were grown and maintained using GAM broth (Nissui Pharmaceuticals) containing $0.1 \%(\mathrm{w} / \mathrm{v})$ sodium thioglycolate as reducing agent, GAM agar containing $0.1 \%(\mathrm{w} / \mathrm{v})$ sodium taurocholate (Nakarai) or PYG broth (containing 3\% trypticase peptone, $2 \%$ yeast extract, $1 \%$ glucose and $0.1 \%$ sodium thioglycolate). Where indicated, GAM broth was buffered by adding $70 \mathrm{mM}$ phosphate buffer to a final $\mathrm{pH}$ of 7.0. Cultures were incubated at $37^{\circ} \mathrm{C}$ in an anaerobic chamber using $10 \% \mathrm{CO}_{2}, 10 \% \mathrm{H}_{2}$ and $\mathrm{N}_{2}$ to balance.

Preparation of spore suspensions. Overnight cultures of $C$. difficile and CBM588 were inoculated at 1:1000 into $40 \mathrm{ml}$ fresh medium. These cultures were incubated for 1 week in an anaerobic chamber at $37^{\circ} \mathrm{C}$. To isolate spores, the cultures were centrifuged at $5000 \mathrm{~g}$ for $10 \mathrm{~min}$ to pellet the cells and washed twice with sterile $0.01 \mathrm{M}$ PBS ( $\mathrm{pH}$ 7.2). The resulting pellet was resuspended in $4 \mathrm{ml}$ sterile PBS and heated to $70{ }^{\circ} \mathrm{C}$ for $10 \mathrm{~min}$ to inactivate vegetative cells. Spores were then counted using dilution plating on GAM agar with $0.1 \%$ taurocholate, and stored at $-30{ }^{\circ} \mathrm{C}$.

Co-culture, supernatant and killed-cell experiments. Routinely, C. difficile and CBM588 overnight cultures were inoculated at 1:1000 into fresh medium for culture experiments. For monoculture experiments, the cell number was ascertained at intervals by dilution plating onto GAM agar containing $0.1 \%$ taurocholate. For co-culture experiments, overnight cultures were diluted $1: 1000$ and $1: 100000$ in fresh medium and inoculated at ratios of $1: 1,1: 100$ and $1: 10000$. For spore co-culture experiments, thawed spore suspensions were inoculated together into GAM broth to a final CBM : C. difficile ratio of $1: 1000$ and then incubated at $37^{\circ} \mathrm{C}$ in an anaerobic chamber as above to allow the spores to germinate. At the time periods indicated, $1 \mathrm{ml}$ samples were taken for a cytotoxicity assay and for Western blotting. For dialysis membrane isolation experiments, CBM588 and C. difficile were inoculated on different sides of a cellulose membrane (Sanko) with a maximum pore size of $14 \mathrm{kDa}$.

Supernatants of $C$. difficile, CBM588 or co-cultures were prepared by centrifuging $48 \mathrm{~h}$ cultures at $5000 \mathrm{~g}$ for $10 \mathrm{~min}$ and filtering through $0.22 \mu \mathrm{m}$ filters (Millipore) to sterilize. Supernatants used for subsequent culture experiments were adjusted to $\mathrm{pH} 7$ using $1 \mathrm{M} \mathrm{NaOH}$ and mixed with fresh medium to the required concentrations before inoculation. The effect of CBM588 on C. difficile toxin was tested by inoculating overnight cultures at 1:1000 into $\mathrm{pH}$-adjusted $48 \mathrm{~h} \mathrm{C}$. difficile cell-free supernatant. Samples were taken at 24,48 and $72 \mathrm{~h}$ for the cytotoxicity assay.
Killed cells of CBM588 were prepared by autoclaving overnight cultures at $105{ }^{\circ} \mathrm{C}$ for $15 \mathrm{~min}$ or by incubating pelleted cells in $2.5 \%$ glutaraldehyde (v/v) at room temperature for $1 \mathrm{~h}$. Pellets were washed three times in sterile PBS. Cell fragments were prepared by beadbeating cell pellets suspended in PBS with $0.1 \mathrm{~mm}$ glass beads in a multibead-beater (Yasui Kikai). The supernatant was sterilized by filtering through a $0.22 \mu \mathrm{m}$ filter (Millipore). Preparations were inoculated into cultures at 0 and $24 \mathrm{~h}$ at $10^{6}-10^{8}$ cells $\mathrm{ml}^{-1}$.

pH-adjustment experiments and HPLC. Buffered culture medium was adjusted to the appropriate $\mathrm{pH}$ using $1 \mathrm{M} \mathrm{NaOH}$ or $1 \mathrm{M} \mathrm{HCl}$. Toxin-inactivation assays were performed using crude toxin isolated by $0.22 \mu \mathrm{m}$ filtration of $48 \mathrm{~h}$ C. difficile supernatant. Crude toxin was adjusted to the required $\mathrm{pH}$ and incubated at $37{ }^{\circ} \mathrm{C}$. Aliquots were withdrawn every $2 \mathrm{~h}$ for the cytotoxic assay. HPLC of organic acid concentrations was performed at various time intervals using an LC6A HPLC system (Shimadzu) with a Shim-pack SCR-102H column and a CCD-6A electroconductivity detector. For this and the growthinhibition experiments, the statistical significance of the results was calculated by a paired-sample $t$-test, with $P<0.05$ being considered significant.

Cytotoxicity assay. The African green monkey kidney cell line Vero S1 has been documented previously as a sensitive indicator of C. difficile toxin activity (Welch et al., 1985). Briefly, cells were maintained in minimum essential medium (Sigma Aldrich) containing $10 \%$ fetal bovine serum (Sigma Aldrich) in flasks or 96-well plates. For the cytotoxicity assay, the cell-culture medium was removed and replaced with fresh cell-culture medium or dilutions of cell-free supernatants (diluted from 1:12.5 to $1: 104857600$ ). Cells were incubated at $37{ }^{\circ} \mathrm{C}$ in $5 \% \mathrm{CO}_{2}$ overnight and viewed under a light microscope. The cytotoxicity of the supernatant was determined as the lowest concentration resulting in $>50 \%$ cell rounding. This value is referred to as the $\mathrm{CD}_{50}$ for the sample. Cytotoxicity experiments were repeated at least three times.

Western blotting. Culture samples $(1 \mathrm{ml})$ for Western blot were centrifuged at $10000 \mathrm{~g}$ for $5 \mathrm{~min}$ to pellet the cells and the supernatant was removed to a fresh tube. The pellet was then washed with $0.01 \mathrm{M}$ PBS to remove excess supernatant and the cells were lysed using $2 \mathrm{mg}$ lysozyme $\mathrm{ml}^{-1}$ at $37^{\circ} \mathrm{C}$ for $1 \mathrm{~h}$. The supernatant was filtered through a $0.22 \mu \mathrm{m}$ filter to remove any remaining bacterial cells. The pellet and supernatant samples were then mixed $1: 1$ with $2 \times$ SDS loading buffer containing DTT (ATTO) and heated for $5 \mathrm{~min}$ in a boiling water bath. Samples were stored at $-30{ }^{\circ} \mathrm{C}$ before use. A $30 \mu \mathrm{l}$ aliquot of each thawed sample was run for $120 \mathrm{~min}$ at $20 \mathrm{~mA}$ on an SDS 5-20\% gradient polyacrylamide gel and then transferred to PVDF membranes for $45 \mathrm{~min}$ at $0.22 \mathrm{~mA}$. Membranes were blocked in $5 \%$ skimmed milk in PBS with $0.1 \%$ Tween 20 overnight. Goat primary antibody to $C$. difficile toxin B was obtained from Techlab. Blocked membranes were incubated with primary antibody at a $1: 10000$ dilution in PBS with $0.1 \%$ Tween 20 for $40 \mathrm{~min}$, followed by secondary horseradish peroxidase-conjugated donkey anti-goat IgG (Santa Cruz Biotechnologies) diluted 1:10000 in PBS with 0.1\% Tween 20 for 30 min, washing with four changes of PBS with $0.1 \%$ Tween 20 for 20 min between each incubation. Bound antibody was visualized using an Amersham ECL Plus Western blotting system (GE Healthcare) according to the manufacturer's protocol.

Real-time PCR. Real-time PCR was used to quantify toxin mRNA levels and to ascertain cell numbers in co-culture experiments, as no selective agar could be found to differentiate the two strains. Primers for toxin A and toxin B were used for both mRNA and cell-number quantification and are shown in Table 1, together with the conditions used for each PCR. RT-PCR has been shown previously to accurately quantify individual species of bacteria in complex co-culture mixes such as faecal samples (Matsuki et al., 2004) and to enumerate 
Table 1. Oligonucleotides used in this study

\begin{tabular}{|llccc|}
\hline Name & \multicolumn{1}{c|}{ Sequence $\left(\mathbf{5}^{\prime} \rightarrow \mathbf{3}^{\prime}\right)$} & Target size $(\mathbf{b p})$ & $\boldsymbol{T}_{\mathbf{m}}\left({ }^{\circ} \mathbf{C}\right)$ & Source \\
\hline TcdA-F & GGGGGATCAGGACACACAGTGAC & 196 & 68.0 & This study \\
TcdA-R & GGAACTGCTCCAGTTTCCCACCA & 156 & 58.0 & Lee \& Song (2005) \\
TcdB-F & CACTTCTTTTCAGCACCATCAA & & 56.7 \\
TcdB-R & TGTCCATCCTGTTTCCCAAG & - & This study \\
TcdA-RTR & AGAGTAAGTTCCTCCTGCTC & - & 50.1 & This study \\
TcdB-RTR & TAGTTACATCTCTCACAACA & & & \\
& & & & \\
\hline
\end{tabular}

${ }^{\star} \mathrm{TcdA}$ and $\mathrm{TcdB}$ primers are specific to the $t c d A$ and $t c d B$ gene loci, respectively. F, Forward primer; R, reverse primer. RTR primers are genespecific primers used for reverse transcription.

C. difficile in place of dilution plating (Dingle et al., 2010). In our preliminary experiments, primers to toxin A and toxin B showed good concordance with dilution-plating results with monocultures in the range $10^{3}-10^{9}$ cells, and template controls using CBM588 DNA showed no cross-reaction (data not shown).

Whole-culture samples $(1 \mathrm{ml})$ were centrifuged at $10000 \mathrm{~g}$ for $5 \mathrm{~min}$ to pellet the cells. Total RNA was isolated from pellets by first treating with RNAprotect Bacteria Reagent (Qiagen) to preserve mRNA and then using a Qiagen RNeasy kit (Qiagen). Total DNA was isolated using a Wizard Genomic DNA Purification kit (Promega) following the manufacturer's instructions. DNA was removed from RNA samples by incubation with Turbo DNAfree (Ambion) for $30 \mathrm{~min}$ at $37^{\circ} \mathrm{C}$. RNA was converted to cDNA using PrimeScript reverse transcriptase (Takara) with gene-specific primers as shown in Table 1. Real-time PCRs were carried out using SYBR Premix DimerEraser (Takara) in an ABI Prism 7500 Real-Time PCR system (Applied Biosystems) for 40 cycles using the conditions in Table 1 and a melting curve. Results were analysed using ABI Sequence Detection Software (version 1.2.1). Serial dilutions of whole DNA isolated from overnight cultures of $C$. difficile were used as controls for mRNA PCRs. Whole DNA isolated from control cultures of known cell number (ascertained by dilution plating) was used as the standard for cell-count PCRs. No-template controls were included in every experiment and each reading was repeated in triplicate. Real-time experiments were repeated at least three times.

\section{RESULTS AND DISCUSSION}

\section{Co-culture causes complete and persistent loss of toxicity}

It is well-documented that $C$. difficile can be inhibited when co-cultured with other gut bacteria. For example, faecal emulsions from well patients inhibit $C$. difficile growth and toxicity in vitro (Borriello \& Barclay, 1986; Yamamoto-Osaki et al., 1994). Previous studies on other probiotics have also reported growth and toxicity inhibition in co-culture with various species of Bifidobacterium and Lactobacillus (Banerjee et al., 2009; Trejo et al., 2010). CBM588 in particular has shown inhibition in co-culture towards other pathogenic bacteria such as Escherichia coli O157 (Takahashi et al., 2004) and H. pylori (Takahashi et al., 2000) and additionally has the effect of supporting the growth of the gut's endemic Lactobacillus species (Ichikawa et al., 1999).
In order to test the effect of co-culture on toxicity, the highly toxigenic C. difficile VPI 10463 strain or the clinical isolate TMG-10 were co-cultured at a ratio of $1: 1$ with CBM588, which resulted in a complete loss of toxicity at $24 \mathrm{~h}$ compared with the monoculture control. To test further the effect of dosage, C. difficile VPI 10463 was cultured with CBM588 at various CBM:C. difficile inoculation ratios from $10000: 1$ to $1: 10000$. Cytotoxicity at $24 \mathrm{~h}$ in coculture was not detected when CBM588 was present at a concentration equal to or in excess of $C$. difficile, and was greatly reduced even if $C$. difficile was in excess (Table 2). This reduction in cytotoxicity continued at $48 \mathrm{~h}$, indicating that it was a long-term effect, and addition of fresh medium at $24 \mathrm{~h}$ had no effect on this inhibition, suggesting that nutrient depletion was not the main cause (data not shown). Western blots showed that there was very little toxin buildup inside the bacterial cells, which mirrored the lack of toxin in the supernatant in these samples (Fig. 1). It was observed that toxin tended to build up inside $C$. difficile cells before release into the supernatant began at a certain time point in the growth phase.

\section{Growth and toxin mRNA in co-culture exhibit a dose-dependent inhibition}

The substances previously implicated in the inhibitory effect by CBM588 were SCFAs, particularly butyric acid, which was shown to be toxic even at a neutral $\mathrm{pH}$. However, as $C$. difficile also produces butyric acid, we did not expect this to be the cause of the inhibition in this case. In fact, previous research on toxin regulation showed that butyrate not only fails to affect growth, but actually increases toxin yield (Karlsson et al., 2003).

Growth of the two strains in co-culture when the number of C. difficile cells was equal to or greater than that of CBM588 showed no growth inhibition for either VPI 10463 or TMG10. However, when CBM588 was in excess of $C$. difficile in the co-culture medium, a degree of inhibition proportional to the inoculation ratio was observed. Inoculation ratios of $100: 1$ and 10000:1 caused growth inhibition of approximately 10 - and 100 -fold, respectively (Fig. 2a). This was similar to the results obtained in a previous mouse-model study where administration of CBM588 resulted in a 10- to 
Table 2. Relative cytotoxicity at different inoculation ratios

The cytotoxicity of various ratios of unbuffered and buffered (approx. pH 5.9) co-cultures are expressed as $\log _{10}$ values relative to an equivalent C. difficile monoculture control. $\mathrm{CD}_{50}$ values for the controls were: $1: 9.01 \times 10^{6}$ at a $1: 1$ ratio of $\mathrm{CBM}:$ C. difficile, $1: 1.52 \times 10^{6}$ at $100: 1$ and $1: 1.63 \times 10^{5}$ at $10000: 1$. ND indicates that no cytotoxicity was detected at the lowest dilution corresponding to a $\log _{10}$ relative cytotoxicity at $48 \mathrm{~h}$ of $<-5.86$ at $1: 1$ and $1: 100,<-5.08$ at $100: 1$ and $<-4.12$ at $10000: 1$. All values shown are means \pm SEM of at least three independent experiments.

\begin{tabular}{|c|c|c|c|c|c|}
\hline \multicolumn{2}{|c|}{ Inoculation ratio } & \multicolumn{4}{|c|}{$\log _{10}$ relative cytotoxicity } \\
\hline \multirow[t]{2}{*}{ CBM588 } & \multirow[t]{2}{*}{ C. difficile } & \multicolumn{2}{|c|}{ Unbuffered } & \multicolumn{2}{|c|}{ Buffered } \\
\hline & & $24 \mathrm{~h}$ & $48 \mathrm{~h}$ & $24 \mathrm{~h}$ & $48 \mathrm{~h}$ \\
\hline 10000 & 1 & ND & ND & $\mathrm{ND}$ & $\mathrm{ND}$ \\
\hline 100 & 1 & ND & $\mathrm{ND}$ & $-0.71 \pm 0.19$ & $-3.51 \pm 0.34$ \\
\hline 1 & 1 & ND & $\mathrm{ND}$ & $-0.06 \pm 0.23$ & $-3.60 \pm 0.40$ \\
\hline 1 & 100 & ND & ND & $0.42 \pm 0.26$ & $-3.14 \pm 0.20$ \\
\hline 1 & 10000 & $-0.60 \pm 0.35$ & $-5.32 \pm 0.10$ & $0.57 \pm 0.19$ & $-2.83 \pm 0.20$ \\
\hline
\end{tabular}

100-fold reduction in C. difficile growth (Kamiya et al., 1997).

However, when spores of both CBM588 and C. difficile were inoculated, a greater inhibitory effect was observed over $24 \mathrm{~h}$. C. difficile viable cell numbers decreased by approximately 100-fold over the initial $9 \mathrm{~h}$ when CBM588 was in the exponential phase, but increased once CBM588 reached the stationary phase to give a 10 -fold difference at $24 \mathrm{~h}$ (Fig. 2b). In contrast to vegetative CBM588, which required a $10000: 1$ inoculation ratio to exhibit this 100-fold

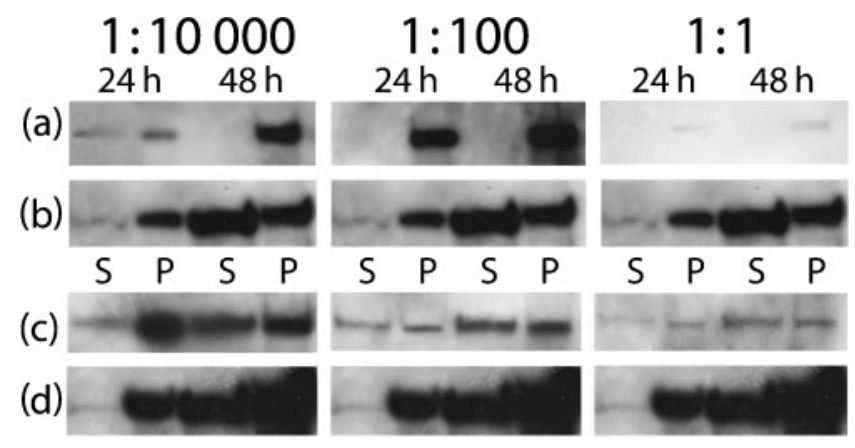

Fig. 1. Western blot showing a decrease in toxin yield of C. difficile compared with the control in co-culture with CBM588. The Western blot shows the toxin B yield from pellet $(P)$ and supernatant $(S)$ at 24 and $48 \mathrm{~h}$ in co-culture. Inoculation ratios are expressed in the form CBM588: C. difficile. Unbuffered GAM medium ( $a, b)$ and GAM medium buffered with $70 \mathrm{mM}$ phosphate buffer to $\mathrm{pH} 7.0$ (c, d) are shown. Toxin yield was significantly reduced in co-culture $(a, c)$ relative to the control $(b, d)$ in all cultures and was dependent on the inoculation ratio; although this effect was still present in buffered medium, it was slightly reduced. It can be observed that, at a certain point in the growth phase, toxin is released from cells causing a decrease in the intensity of the pellet band. The $C$. difficile cell number was the same in all co-cultures compared with the controls. growth inhibition, CBM588 spores not only achieved this after $9 \mathrm{~h}$, but reduced the number of viable $C$. difficile spores in the culture medium. This occurred even at low inoculation ratios of $1: 1000$, suggesting that low doses of CBM588 spores could inhibit $C$. difficile germination successfully in the gut. From these results, it appeared that CBM588 was more efficient at inhibiting the growth of spores or preventing spore germination than the growth of vegetative cells, and that the recovery of $C$. difficile cell numbers after $9 \mathrm{~h}$ could be attributed to the increased proportion of vegetative $C$. difficile in the medium. The inhibitory effect could in this case be caused by the large quantities of organic acids secreted by CBM588. As CBM588 germinates and doubles at nearly twice the rate of C. difficile (K. Oka \& M. Takahashi, unpublished data), it will start to secrete organic acids while $C$. difficile is still germinating. Organic acids such as vinegar and lemon juice are already known to inhibit spore germination in Clostridium perfringens (Valenzuela-Martinez et al., 2010), so we would expect butyric acid to have a similar effect on C. difficile spore germination in this case.

Toxin mRNA levels have been shown to respond to a variety of environmental signals such as temperature, nutrient concentrations and antibiotics (Karlsson et al., 1999, 2003; Yamakawa et al., 1998; Nakamura et al., 1982). We checked for the downregulation of toxin mRNAs by nutrient depletion or other signalling molecules using real-time PCR. However, mRNA levels of $t c d A$ and $t c d B$ transcripts during co-culture showed only a small reduction, consistent with the growth inhibition observed during the experiment. Both transcript levels decreased by approximately 50-fold and 5000-fold in $100: 1$ and 10000: 1 culture ratios, respectively, relative to an equivalent $C$. difficile monoculture control (Table 3). As mRNA expression is heavily dependent on growth phase (Hundsberger et al., 1997), the greater degree of growth inhibition in the 10000:1 culture would account for the slower production of toxin mRNA in these samples. Interestingly, both $t c d A$ and $t c d B$ transcripts were normal or 

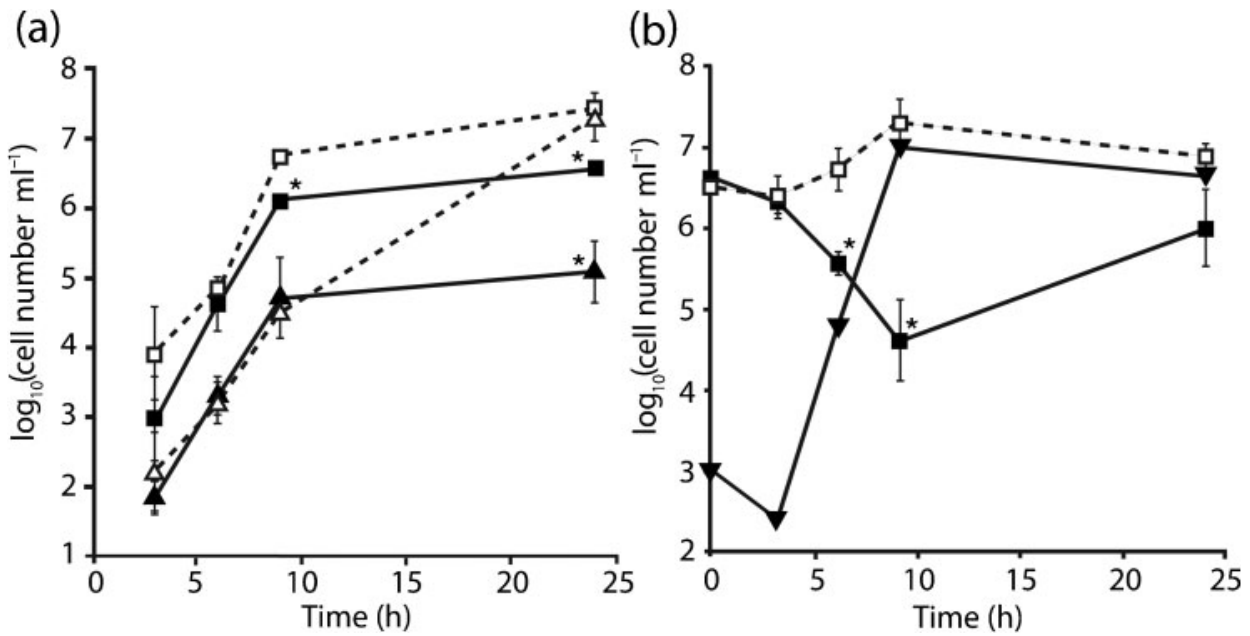

Fig. 2. Co-culture with CBM588 causes mild dose-dependent growth inhibition of $C$. difficile. Dose-dependent growth inhibition of $C$. difficile was observed in co-culture with both vegetative cells (a) and spores (b) of CBM588. In the case of vegetative cells, there was no difference at a $1: 1$ (CBM : C. difficile) ratio (data not shown). At $24 \mathrm{~h}$, at $100: 1$ ( $\boldsymbol{\square}$, co-culture; $\square$, control), approximately tenfold inhibition was seen, whilst at $10000: 1$ ( $\boldsymbol{\Lambda}$, co-culture; $\triangle$, control), approximately 100-fold inhibition was seen. In the case of spores ( $\mathbf{\square}$, co-culture; $\square$, control), a greater inhibition was seen, even at a low inoculation ratio of $1: 1000$, where $C$. difficile declined over the initial $9 \mathrm{~h}$ while CBM588 $(\boldsymbol{\nabla})$ was in the exponential phase. Results shown are means \pm SEM of at least three independent experiments. ${ }^{*}$ Statistically significant decrease compared with the control $(P<0.05)$.

increased by up to 30 -fold relative to the control if $C$. difficile was equal to or in excess of CBM588, even though the cell counts of $C$. difficile in these cultures were equivalent to the control monocultures (Fig. 2a), which we ascribed to upregulation by increased butyric acid in the medium. Therefore, it was clear that the reduction in cytotoxicity occurred as a result of the lack of mature toxin and was not caused by a significant reduction in gene transcription.

\section{Inhibition of toxicity in co-culture appears to require cell contact}

Previous studies have suggested that probiotics may release substances into the supernatant that inhibit toxin production or adhesion to colonocytes (Banerjee et al., 2009) or that destroy mature toxin (Castagliuolo et al., 1999). One study also considered the role of quorum sensing, although this has been shown to have at most a minor effect on toxin production (Lee \& Song, 2005; Carter et al., 2005), and our preliminary studies found very low LuxS/AI-2 activity in CBM588 (data not shown).

However, pH-neutral CBM588 supernatant was found to have no effect on the growth of the TMG-10, KY-8, KY-34, KY-104 and SLC clinical isolates of C. difficile or the reference strain VPI 10463 (data not shown) and only a slight effect on toxicity. When compared with the effect of supernatant isolated from the non-toxigenic KZ-1692 strain of $C$. difficile, there was no significant difference

Table 3. Relative toxin mRNA levels at different inoculation ratios

mRNA values are shown relative to an equivalent $C$. difficile monoculture control. Absolute mean mRNA quantity values for tcdA controls were $4.23 \times 10^{4}$ for a ratio of $1: 1$ and below of CBM: C. difficile, $8.61 \times 10^{4}$ for $100: 1$ and $4.92 \times 10^{5}$ for $10000: 1$; and for $t c d B$ were $2.01 \times 10^{4}$ for $1: 1$ and below, $3.13 \times 10^{4}$ for $100: 1$ and $1.89 \times 10^{5}$ for $10000: 1$. All values shown are the mean of three independent experiments. The lowest and highest values observed are shown in parentheses. Growth data for this experiment are shown in Fig. 2(a).

\begin{tabular}{|llccc|}
\hline \multicolumn{2}{c}{ Inoculation ratio } & & \multicolumn{2}{c|}{ Relative mRNA level at 24 h } \\
\cline { 1 - 1 } CBM588 & C. difficile & & cddA & tcdB \\
\hline 10000 & 1 & & $2.83 \times 10^{-4}\left(1.92-3.75 \times 10^{-4}\right)$ & $4.55 \times 10^{-3}\left(1.44-7.65 \times 10^{-3}\right)$ \\
100 & 1 & $0.04(0.03-0.04)$ & $0.14(0.03-0.18)$ \\
1 & 1 & $13.66(13.0-14.6)$ & $6.08(2.4-13.3)$ \\
1 & 100 & $29.74(10.1-42.0)$ & $7.37(4.4-15.0)$ \\
1 & 10000 & $2.99(1.1-14.1)$ & $9.15(1.0-16.5)$ \\
\hline
\end{tabular}


(Fig. 3). In this case, the difference in toxicity was likely to be due to nutrient depletion, as $C$. difficile is only able to metabolize simple nutrients and we would therefore expect that the use of a depleted cell-free supernatant would impair its metabolism.

Culture of the two strains separated by a permeable cellulose membrane (pore size $<14 \mathrm{kDa}$ ) also showed no inhibition of $C$. difficile toxicity. In order to confirm whether the presence of $C$. difficile cells in the CBM588 culture affected the secretion of an inhibitor substance, the experiment was repeated using a co-culture of CBM588 and C. difficile VPI 10463 instead of CBM588 monoculture (clostridial toxins are much larger than the membrane cutoff and do not pass through the dialysis tubing). However, this too showed normal toxicity. This suggests strongly that the co-culture inhibitory effect is not governed by a secreted molecule and cannot be due to nutrient depletion, as simple nutrients diffuse freely through the membrane.

This led us to hypothesize that the effector molecule may be present on the cell surface of CBM588, but attempts to elicit the same response with CBM588 cells killed by glutaraldehyde or heat, or on cells fragmented by bead beating, were unsuccessful. However, these methods greatly denature the membrane proteins, and filtration of cell fragments may result in retention of most of the cell material. Unfortunately, no other more suitable methods for testing this were available to us at the time.

Studies on the probiotic yeast $S$. boulardii have found that it inhibits $C$. difficile toxin in vitro by secreting a protease that degrades the mature protein (Castagliuolo et al., 1999) and also by modulating the gut immune response (Chen et al.,
2006; Kyne et al., 2001). However, data on the recently sequenced genome of CBM588 show that, although it contains protease genes, none of them is confirmed as being secreted (unpublished data). Accordingly, we found that neither live CBM588 nor its cell-free supernatant had a detoxifying effect on sterile samples of $C$. difficile toxin. CBM588 has shown beneficial effects on colonic inflammation in a rat dextran sulphate colitis model, reportedly by increased production of SCFAs in the colon (Okamoto et al., 2000) and enhancement of host Lactobacillus species (Ichikawa et al., 1999). It is therefore possible that this effect accounts partly for the previous in vivo results, but it would not account for the observations in this study.

We concluded from this that inhibition of toxicity is not governed by a secreted factor, as with many other probiotics, but appears to rely instead on an as-yet-uncharacterized cell-cell interaction between the two species.

\section{Low-pH conditions inhibit C. difficile growth and toxicity}

C. difficile is highly adapted to the human gut environment and, as such, $48 \mathrm{~h}$ cultures of $C$. difficile generally exhibit $\mathrm{pH}$ values of around 6.2, similar to the gut $\mathrm{pH}$. Similar cultures of CBM588 have a much lower $\mathrm{pH}$ of around 4.9, with co-cultures generally exhibiting a $\mathrm{pH}$ of 4.9-5.2. This low $\mathrm{pH}$ is caused by the large quantity of organic acids produced by this strain. SCFAs such as butyrate also act as an energy source for colonocytes and have been shown to be beneficial in other forms of colitis (Scheppach et al., 1992), as well as in the possible prevention of colon cancer (McIntyre et al., 1993; Scharlau et al., 2009). (a)

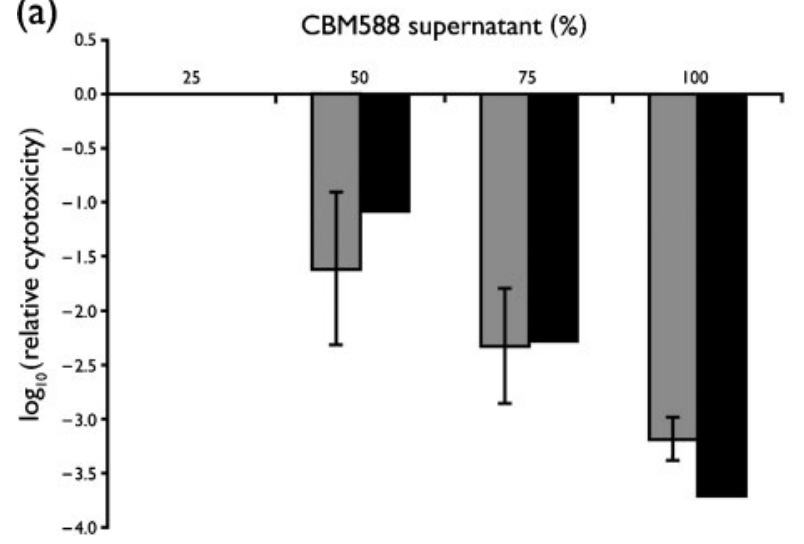

(b)

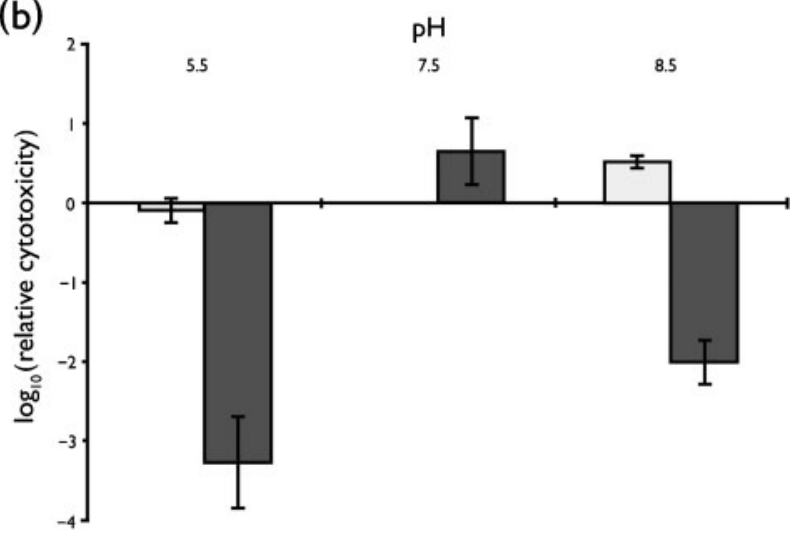

Fig. 3. Effect of CBM588 cell-free supernatant and $\mathrm{pH}$ on C. difficile toxicity. (a) The effect of various concentrations of $\mathrm{pH}-$ adjusted CBM588 cell-free supernatant at $48 \mathrm{~h}$ (shaded bars) is expressed as mean $\log _{10}$ values \pm SEM relative to a $0 \%$ control (no added supernatant). The equivalent mean result (filled bars) of the supernatant of the non-toxigenic strain $\mathrm{C}$. difficile $\mathrm{KZ}-$ 1692 was used for comparison. The $C_{50}$ values of the control cultures were $1: 800$ at $24 \mathrm{~h}$ and $1: 3.28 \times 10^{6}$ at $48 \mathrm{~h}$. (b) The effect of $\mathrm{pH}$ on C. difficile monoculture at $24 \mathrm{~h}$ (light grey bars) and $48 \mathrm{~h}$ (dark grey bars) is expressed as mean $\log _{10}$ values \pm SEM relative to $\mathrm{pH} 6.5$. The Western blot of $\mathrm{pH}$-adjusted cultures is shown in Fig. $4(\mathrm{c})$. The $\mathrm{CD}_{50}$ values of the control cultures were $1: 800$ at $24 \mathrm{~h}$ and $1: 2.46 \times 10^{6}$ at $48 \mathrm{~h}$. All values shown are the mean of three independent experiments. 
We measured the growth and toxicity of $C$. difficile VPI 10463 strain monocultures at a $\mathrm{pH}$ range of $4.5-8.5$ to assess the effect of $\mathrm{pH}$ on this strain, as it exhibits the greatest toxin production, facilitating comparison by Western blotting. Growth of $C$. difficile at $\mathrm{pH} 4.5$ was negligible (not shown) and growth at $\mathrm{pH} 5.5$ was markedly reduced, although it reached the same level as the control at $\mathrm{pH} 6.5$ after $24 \mathrm{~h}$ (Fig. 4a). Growth at $\mathrm{pH} \geqslant 6.5$ was normal. HPLC measurement of organic acid production at $\mathrm{pH} 5.5$ found a significant 8 -fold reduction in total acids produced at $24 \mathrm{~h}$ compared with the control at $\mathrm{pH} 6.5$ (Fig. 4b). Toxin production as measured by Western blotting (Fig. 4c) and a cytotoxic assay (Fig. 3) showed that intracellular toxin levels at $48 \mathrm{~h}$ were highest at $\mathrm{pH}$ 7.5.

C. difficile toxin B was shown previously to require an acidic endosome for activity during endocytosis and it was found that extracellular low $\mathrm{pH}$ can substitute for this effect (Qa'Dan et al., 2000). Results from the same study also indicated that maintenance of toxin in a low-pH environment reduced its cytotoxicity, although it was not clear whether this was a reversible effect. We observed that raw $C$. difficile toxin $B$ in a cell-free supernatant incubated at $37{ }^{\circ} \mathrm{C}$, at a $\mathrm{pH}$ of $<6.0$, decreased steadily in cytotoxicity (Fig. 4d), which may be due to irreversible conformational changes of the toxin B molecule, causing it to be unable to bind to membrane receptors. Western blotting showed band widening and blurring, possibly due to degradation of the misfolded toxin (Fig. 4e).
We concluded that the low $\mathrm{pH}$ in co-cultures probably had a significant effect on both the growth and the observed efficacy of toxin B. This may also have affected previous studies on Lactobacillus and Bifidobacterium as these strains also produce organic acids and it was not clear whether the $\mathrm{pH}$ was controlled in these studies.

\section{Toxin inhibition persists at colonic pH}

In order to control for $\mathrm{pH}$ changes during co-culture, GAM broth was buffered with $70 \mathrm{mM}$ phosphate buffer at $\mathrm{pH}$ 7.0. This was the highest concentration of potassium tolerated by both strains with no growth inhibition. Over $24 \mathrm{~h}$, the $\mathrm{pH}$ of the buffered medium decreased to $5.8-5.9$ and was maintained at this level. This is similar to the $\mathrm{pH}$ in the large intestine in humans, which ranges from 5.6 to 6.8 (Wilson, 2005). In this buffered environment, coculture excess of CBM588 at 100:1 and 10 000:1 ratio inoculations continued to exhibit little or no toxin production at 24 and $48 \mathrm{~h}$. When C. difficile was inoculated at the same level or in excess relative to CBM588 (ratios of $1: 1,1: 100$ and $1: 10000$ ), supernatants at $24 \mathrm{~h}$ showed equal or slightly increased cytotoxicity relative to controls, consistent with an increase in toxin production due to a combination of butyric acid concentration and a higher starting $\mathrm{pH}$. However, supernatants at $48 \mathrm{~h}$ showed a stark reduction in relative cytotoxicity, and this was supported by a reduction in toxin yield as shown by Western blotting. At ratios of $1: 1,1: 100$ and $1: 10000$, (a)

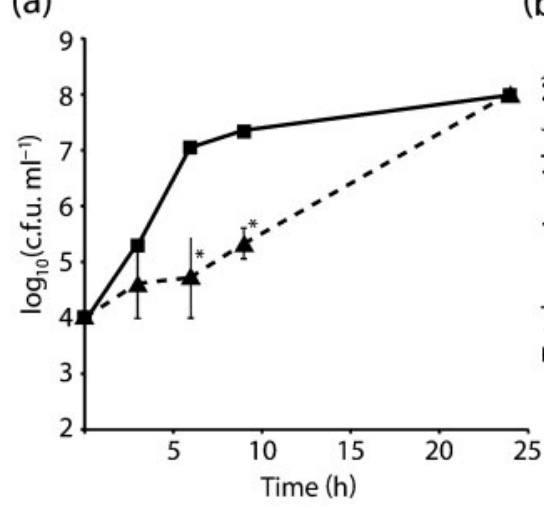

(c)

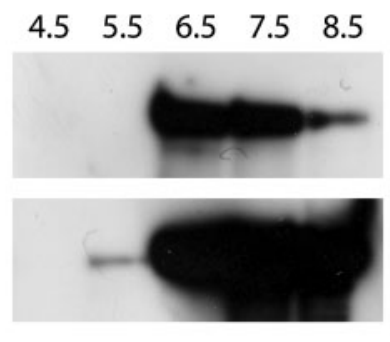

$24 \mathrm{~h}$

$48 \mathrm{~h}$

(e)

$0 \mathrm{~h} \quad 4 \mathrm{~h} \quad 8 \mathrm{~h} \quad 24 \mathrm{~h}$

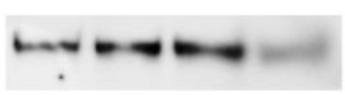

(b)

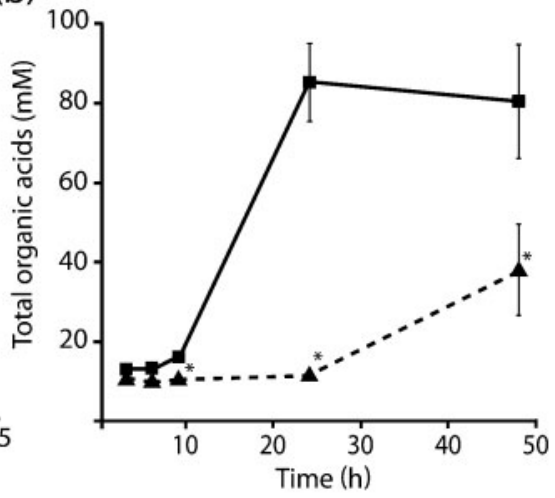

(d)

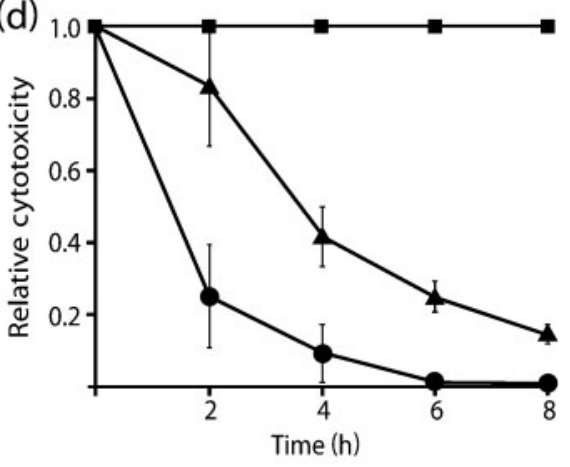

Fig. 4. Low $\mathrm{pH}$ decreases $C$. difficile growth and metabolism but also toxin $\mathrm{B}$ activity. The effect of an acidic $\mathrm{pH}$ on growth (a), metabolism (expressed by production of organic acids) (b) and toxin B production (c) is shown, together with the decrease in toxin activity in cell-free culture at acidic $\mathrm{pH}(\mathrm{d}, \mathrm{e})$. (a) Monoculture growth at $\mathrm{pH} 5.5(\mathbf{\Lambda})$ was slower than at $\mathrm{pH} 6.5$ ( $\mathbf{\square})$ but reached the same level after $24 \mathrm{~h}$. (b) Production of organic acids at $\mathrm{pH} 5.5$ ( $\mathbf{\Delta})$ was much slower than at $\mathrm{pH} 6.5$ (ם) and did not reach the same level, even after $48 \mathrm{~h}$ of growth. *Statistically significant decrease compared with the control $(P<0.05)$. (c) Toxin B production, as measured by Western blotting, was greatest between $\mathrm{pH} 6.5$ and 7.5 after $48 \mathrm{~h}$. (d) Toxin isolates incubated at $37{ }^{\circ} \mathrm{C}$ at a pH of $<6.0$ lost activity steadily over $8 \mathrm{~h}$. Activity was lost more quickly at the lower $\mathrm{pHs}$ of $5.0(\mathbf{O})$ and $5.5(\mathbf{A})$, whereas a $\mathrm{pH}$ of $\geqslant 6.0$ or inoculation of CBM588 cells $(\boldsymbol{\square})$ had no effect. Results shown are the means \pm SEM of three independent experiments. (e) Western blots of samples of sterile toxin B incubated at $\mathrm{pH} 5.5$ showing increased band widening and blurring over time. 
toxicity was decreased by 4000-, 1300- and 700-fold, respectively (Table 2; Fig. 1).

This result showed that the effect of low $\mathrm{pH}$ did not account for the previously observed inhibition, although it did have a synergistic effect. The exact mechanism of inhibition was not clarified by our investigation, but we were able to conclude that CBM588 appeared to interfere with protein production in $C$. difficile and the mechanism appeared to require cell contact. As the supernatant alone had no effect in our study, if a secreted factor is responsible, it would have to be both short-lived and $<14 \mathrm{kDa}$, or be active only at acidic $\mathrm{pH}$. It was also observed that toxin was released into the medium earlier in some co-cultures, although intracellular toxin levels did not build up afterwards as in the controls. As toxin is thought to be released via a holing-endolysin reaction by TcdE (Tan et al., 2001), it is tempting to postulate that CBM588 may speed up membrane permeabilization before toxin maturation occurs. However, further research is required to elucidate which, if any, of these is the case.

\section{Conclusions}

The action of CBM588 in vitro was shown not to occur by previously documented methods of growth inhibition, mRNA downregulation or extracellular toxin inactivation; instead, cell contact in co-culture appeared to affect $C$. difficile toxin protein production, which may represent a novel inhibitory mechanism.

From the results of this study, it is clear that the commercially available spore preparation of CBM588 would work best as a prophylactic medication for CDAD, as both growth and toxin inhibition work better at higher inoculation ratios. Additionally, we have shown that even low quantities of CBM588 spores are capable of inhibiting the germination of much larger quantities of $C$. difficile. There are also clinical benefits in the use of a probiotic such as CBM588 over currently used preparations of Lactobacillus and Bifidobacterium species. As it is not feasible to refrigerate all doses of a probiotic before giving them to patients, an often-cited reason for non-compliance is the taste of the yoghurt-drink preparations of these bacteria. As CBM588 is only available as spores in tablet form, this would not be a problem and doses would be easier to measure. To date, CBM588 has an excellent safety record, although cases of probiotic infections are generally very rare (Boyle et al., 2006). Being able to sporulate also allows CBM588 to resist the action of antibiotics that would inhibit other probiotic strains. In terms of cost, a 20 day supply of CBM588 costs approximately $\mathfrak{E} 20$, which is comparable to other commercial probiotics, and it has a far longer shelf-life at room temperature.

Although probiotics continue to be used in the management of CDAD, their mechanism of action remains poorly understood. This, combined with the dizzying array of probiotic strains and preparations available, makes formulation and assessment of treatment regimens a challenging task. More studies investigating the interactions of probiotic strains in combination with larger cohort trials are required before clinicians are able to decide in which cases they are suitable and which probiotics to prescribe.

\section{ACKNOWLEDGEMENTS}

This research was completed under a research scholarship provided by MEXT: The Ministry of Education, Culture, Sports, Science and Technology for Japan.

\section{REFERENCES}

Banerjee, P., Merkel, G. J. \& Bhunia, A. K. (2009). Lactobacillus delbrueckii ssp. bulgaricus B-30892 can inhibit cytotoxic effects and adhesion of pathogenic Clostridium difficile to Caco-2 cells. Gut Pathog 1, 8 .

Borriello, S. P. \& Barclay, F. E. (1986). An in-vitro model of colonisation resistance to Clostridium difficile infection. J Med Microbiol 21, 299-309.

Boyle, R. J., Robins-Browne, R. M. \& Tang, M. L. K. (2006). Probiotic use in clinical practice: what are the risks? Am J Clin Nutr 83, 1256 1264, quiz 1446-1447.

Carter, G. P., Purdy, D., Williams, P. \& Minton, N. P. (2005). Quorum sensing in Clostridium difficile: analysis of a luxS-type signalling system. J Med Microbiol 54, 119-127.

Castagliuolo, I., Riegler, M. F., Valenick, L., LaMont, J. T. \& Pothoulakis, C. (1999). Saccharomyces boulardii protease inhibits the effects of Clostridium difficile toxins $\mathrm{A}$ and $\mathrm{B}$ in human colonic mucosa. Infect Immun 67, 302-307.

Chen, X., Kokkotou, E. G., Mustafa, N., Bhaskar, K. R., Sougioultzis, S., O'Brien, M., Pothoulakis, C. \& Kelly, C. P. (2006). Saccharomyces boulardii inhibits ERK1/2 mitogen-activated protein kinase activation both in vitro and in vivo and protects against Clostridium difficile toxin A-induced enteritis. J Biol Chem 281, 24449-24454.

deVos, P., Garrity, G., Jones, D., Krieg, N. R., Ludwig, W., Rainey, F. A., Schleifer, K.-H. \& Whitman, W. B. (editors) (2009). Bergey's Manual of Systematic Bacteriology, vol. 3. New York: Springer-Verlag.

Dingle, T., Mulvey, G. L., Humphries, R. M. \& Armstrong, G. D. (2010). A real-time quantitative PCR assay for evaluating Clostridium difficile adherence to differentiated intestinal Caco-2 cells. J Med Microbiol 59, 920-924.

Engelbrektson, A., Korzenik, J. R., Pittler, A., Sanders, M. E., Klaenhammer, T. R., Leyer, G. \& Kitts, C. L. (2009). Probiotics to minimize the disruption of faecal microbiota in healthy subjects undergoing antibiotic therapy. J Med Microbiol 58, 663-670.

Henrich, T. J., Krakower, D., Bitton, A. \& Yokoe, D. S. (2009). Clinical risk factors for severe Clostridium difficile-associated disease. Emerg Infect Dis 15, 415-422.

Huang, H., Weintraub, A., Fang, H. \& Nord, C. E. (2009). Antimicrobial resistance in Clostridium difficile. Int $J$ Antimicrob Agents 34, 516-522.

Hundsberger, T., Braun, V., Weidmann, M., Leukel, P., Sauerborn, M. \& von Eichel-Streiber, C. (1997). Transcription analysis of the genes $t c d A-E$ of the pathogenicity locus of Clostridium difficile. Eur $J$ Biochem 244, 735-742.

Ichikawa, H., Kuroiwa, T., Inagaki, A., Shineha, R., Nishihira, T., Satomi, S. \& Sakata, T. (1999). Probiotic bacteria stimulate gut epithelial cell proliferation in rat. Dig Dis Sci 44, 2119-2123. 
Imase, K., Takahashi, M., Tanaka, A., Tokunaga, K., Sugano, H., Tanaka, M., Ishida, H., Kamiya, S. \& Takahashi, S. (2008). Efficacy of Clostridium butyricum preparation concomitantly with Helicobacter pylori eradication therapy in relation to changes in the intestinal microbiota. Microbiol Immunol 52, 156-161.

Kamiya, S., Taguchi, H., Yamaguchi, H., Osaki, T., Takahashi, M. \& Nakamura, S. (1997). Bacterioprophylaxis using Clostridium butyricum for lethal caecitis by Clostridium difficile in gnotobiotic mice. Rev Med Microbiol 8 (Suppl. 1), S60.

Karlsson, S., Burman, L. G. \& Akerlund, T. (1999). Suppression of toxin production in Clostridium difficile VPI 10463 by amino acids. Microbiology 145, 1683-1693.

Karlsson, S., Dupuy, B., Mukherjee, K., Norin, E., Burman, L. G. \& Akerlund, T. (2003). Expression of Clostridium difficile toxins A and B and their sigma factor $\mathrm{TcdD}$ is controlled by temperature. Infect Immun 71, 1784-1793.

Kyne, L., Warny, M., Qamar, A. \& Kelly, C. P. (2001). Association between antibody response to toxin $\mathrm{A}$ and protection against recurrent Clostridium difficile diarrhoea. Lancet 357, 189-193.

Lee, A. S. \& Song, K. P. (2005). LuxS/autoinducer-2 quorum sensing molecule regulates transcriptional virulence gene expression in Clostridium difficile. Biochem Biophys Res Commun 335, 659-666.

Matsuki, T., Watanabe, K., Fujimoto, J., Kado, Y., Takada, T., Matsumoto, K. \& Tanaka, R. (2004). Quantitative PCR with 16S rRNA-gene-targeted species-specific primers for analysis of human intestinal bifidobacteria. Appl Environ Microbiol 70, 167-173.

McFarland, L. V. (2009). Evidence-based review of probiotics for antibiotic-associated diarrhea and Clostridium difficile infections. Anaerobe 15, 274-280.

McFarland, L. V. (2010). Systematic review and meta-analysis of Saccharomyces boulardii in adult patients. World J Gastroenterol 16, 2202-2222.

McIntyre, A., Gibson, P. R. \& Young, G. P. (1993). Butyrate production from dietary fibre and protection against large bowel cancer in a rat model. Gut 34, 386-391.

Nakamura, S., Nakashio, S., Inamatsu, T., Nishida, N., Taniguchi, N. \& Nishida, S. (1980). Toxigenicity of Clostridium difficile isolates from patients and healthy adults. Microbiol Immunol 24, 995-997.

Nakamura, S., Mikawa, M., Tanabe, N., Yamakawa, K. \& Nishida, S. (1982). Effect of clindamycin on cytotoxin production by Clostridium difficile. Microbiol Immunol 26, 985-992.

Nakanishi, S. \& Tanaka, M. (2010). Sequence analysis of a bacteriocinogenic plasmid of Clostridium butyricum and expression of the bacteriocin gene in Escherichia coli. Anaerobe 16, 253-257.

Okamoto, T., Sasaki, M., Tsujikawa, T., Fujiyama, Y., Bamba, T. \& Kusunoki, M. (2000). Preventive efficacy of butyrate enemas and oral administration of Clostridium butyricum M588 in dextran sodium sulfate-induced colitis in rats. J Gastroenterol 35, 341-346.

Pépin, J., Valiquette, L., Alary, M. E., Villemure, P., Pelletier, A., Forget, K., Pépin, K. \& Chouinard, D. (2004). Clostridium difficileassociated diarrhea in a region of Quebec from 1991 to 2003: a changing pattern of disease severity. CMAJ 171, 466-472.

Preidis, G. A. \& Versalovic, J. (2009). Targeting the human microbiome with antibiotics, probiotics, and prebiotics: gastroenterology enters the metagenomics era. Gastroenterology 136, 2015-2031.
Qa'Dan, M., Spyres, L. M. \& Ballard, J. D. (2000). pH-induced conformational changes in Clostridium difficile toxin B. Infect Immun 68, 2470-2474.

Scharlau, D., Borowicki, A., Habermann, N., Hofmann, T., Klenow, S., Miene, C., Munjal, U., Stein, K. \& Glei, M. (2009). Mechanisms of primary cancer prevention by butyrate and other products formed during gut flora-mediated fermentation of dietary fibre. Mutat Res 682, 39-53.

Scheppach, W., Sommer, H., Kirchner, T., Paganelli, G. M., Bartram, P., Christl, S., Richter, F., Dusel, G. \& Kasper, H. (1992). Effect of butyrate enemas on the colonic mucosa in distal ulcerative colitis. Gastroenterology 103, 51-56.

Segarra-Newnham, M. (2007). Probiotics for Clostridium difficileassociated diarrhea: focus on Lactobacillus rhamnosus GG and Saccharomyces boulardii. Ann Pharmacother 41, 1212-1221.

Seki, H., Shiohara, M., Matsumura, T., Miyagawa, N., Tanaka, M., Komiyama, A. \& Kurata, S. (2003). Prevention of antibioticassociated diarrhea in children by Clostridium butyricum MIYAIRI. Pediatr Int 45, 86-90.

Takahashi, M., Taguchi, H., Yamaguchi, H., Osaki, T. \& Kamiya, S. (2000). Studies of the effect of Clostridium butyricum on Helicobacter pylori in several test models including gnotobiotic mice. $\mathrm{J} \mathrm{Med}$ Microbiol 49, 635-642.

Takahashi, M., Taguchi, H., Yamaguchi, H., Osaki, T., Komatsu, A. \& Kamiya, S. (2004). The effect of probiotic treatment with Clostridium butyricum on enterohemorrhagic Escherichia coli O157: H7 infection in mice. FEMS Immunol Med Microbiol 41, 219-226.

Tan, K. S., Wee, B. Y. \& Song, K. P. (2001). Evidence for holin function of $t c d E$ gene in the pathogenicity of Clostridium difficile. J Med Microbiol 50, 613-619.

Trejo, F. M., Pérez, P. F. \& De Antoni, G. L. (2010). Co-culture with potentially probiotic microorganisms antagonises virulence factors of Clostridium difficile in vitro. Antonie van Leeuwenhoek 98, 1929.

Valenzuela-Martinez, C., Pena-Ramos, A., Juneja, V. K., Korasapati, N. R., Burson, D. E. \& Thippareddi, H. (2010). Inhibition of Clostridium perfringens spore germination and outgrowth by buffered vinegar and lemon juice concentrate during chilling of ground turkey roast containing minimal ingredients. J Food Prot 73, 470-476.

Welch, A. R., Borriello, S. P. \& Barclay, F. E. (1985). Simplified procedure for tissue culture in routine detection of cytotoxins. J Clin Pathol 38, 835-837.

Wilcox, M. H., Cunniffe, J. G., Trundle, C. \& Redpath, C. (1996). Financial burden of hospital-acquired Clostridium difficile infection. $J$ Hosp Infect 34, 23-30.

Wilson, M. (2005). Microbial Inhabitants of Humans: Their Ecology and Role in Health and Disease. Cambridge: Cambridge University Press.

Yamakawa, K., Karasawa, T., Ohta, T., Hayashi, H. \& Nakamura, S. (1998). Inhibition of enhanced toxin production by Clostridium difficile in biotin-limited conditions. J Med Microbiol 47, 767-771.

Yamamoto-Osaki, T., Kamiya, S., Sawamura, S., Kai, M. \& Ozawa, A. (1994). Growth inhibition of Clostridium difficile by intestinal flora of infant faeces in continuous flow culture. J Med Microbiol 40, 179187. 